\title{
THE OPERATION AND MANAGEMENT OF A CASE AFTER DIVERSION OF THE INFERIOR VENA CAVA INTO THE LEFT ATRIUM AFTER THE OPEN REPAIR OF AN ATRIAL SEPTAL DEFECT
}

\author{
BY \\ V. O. BJÖRK,* L. JOHANSSON, B. JONSSON, O. NORLANDER, AND B. NORDENSTRÖM \\ From the Chest Unit, Karolinska Sjukhuset, Stockholm, Sweden
}

(RECEIVED FOR PUBLICATION APRIL 10, 1958)

In some cases of atrial septal defect of the secundum type the defect is not localized to the area of the foramen ovale but also involves the dorsal and lower part of the septum in the vicinity of the inferior vena cava (Bedford, Sellors, Somerville, Belcher, and Besterman, 1957; Lewis, Varco, and Taufic, 1954). There is no lower margin to the defect above the orifice of the inferior vena cava, which therefore appears to communicate directly with the left atrium as well as with the right, and, associated with the left-to-right inter-atrial shunt, a small right-to-left shunt, from the inferior vena cava to the left atrium, may be found. From the surgical point of view it is important to recognize this type of defect because its repair may cause a severe complication. Anteriorly the defect has an inferior edge of atrial septum which is continuous with the valve of the inferior vena cava, situated to the right of the orifice. If this valve is wrongly taken as the lower margin of the defect and used when repairing the defect, the inferior vena cava would then be connected to the left atrium and not to the right. Such an error has been made in other clinics (personal communications) utilizing the closed technique of atrio-septopexi of Bailey, and it has occurred in at least five cases operated on under hypothermia. The error has also been made in a case operated upon using extracorporeal circulation where the tube draining the inferior vena cava was inserted through the saphenous vein.

Diversion of the blood from the inferior vena cava into the left atrium is a serious complication. The majority of patients in which the mistake has not been corrected have died on the table. In one case operated on under hypothermia the error was detected immediately on closing the right atrium. Therefore, after a short time a second inflow-occlu-

* Now thoracic surgeon in chief, University Hospital, Upsala, Sweden. sion was made and the defect opened and closed again on the proper side of the inferior vena cava. The patient died. These cases are not reported in the literature.

The aim of this paper is to emphasize this type of atrial septal defect and the error we made in one such case operated upon under hypothermia, and to describe the successful management of this complication.

In our clinic we elected to rewarm the patient, and, after confirmation of the diagnosis by angiocardiography performed from the saphenous vein, six hours later on the same afternoon we immersed the patient in ice water for the second time and operated again, this time successfully.

\section{CASE REPORT}

A 31-year-old woman, who had had two children, had noticed dyspnoea and palpitations on exertion for years. The physical findings were typical of an isolated atrial septal defect. The E.C.G. showed slight right ventricular hypertrophy. The physical working capacity was $550 \mathrm{~kg} . / \mathrm{min}$., corresponding to $70 \%$ of the predicted normal value. The heart volume was $130 \%$ of the predicted normal value. Cardiac catheterization showed a left-to-right interatrial shunt of $12.81 . / \mathrm{min}$. at rest. The pulmonary flow was $17.3 \mathrm{l} . / \mathrm{min}$. (body surface area= $\left.1.48 \mathrm{~m} .{ }^{2}\right)$. The arterial oxygen saturation was $95 \%$. During exercise, at a pulse rate of $150 / \mathrm{min}$. (load= $400 \mathrm{~kg}$. $/ \mathrm{min}$.), the pulmonary flow was unchanged, but the arterial oxygen saturation decreased to $84 \%$. The pressure in the pulmonary artery at rest was $31 / 11 \mathrm{~mm}$. $\mathrm{Hg}($ mean $=21)$ and rose during exercise to $62 / 40 \mathrm{~mm}$. Hg. Angiocardiography was performed with a contrast injection into the left atrium demonstrating a big atrial septal defect of about $4 \frac{1}{2} \mathrm{~cm}$. diameter and displacement of the inferior vena cava posteriorly and medially. It was not possible to visualize any remnant of the atrial septum inferiorly, as there was a considerable reflux of contrast material into the inferior vena cava. This is 


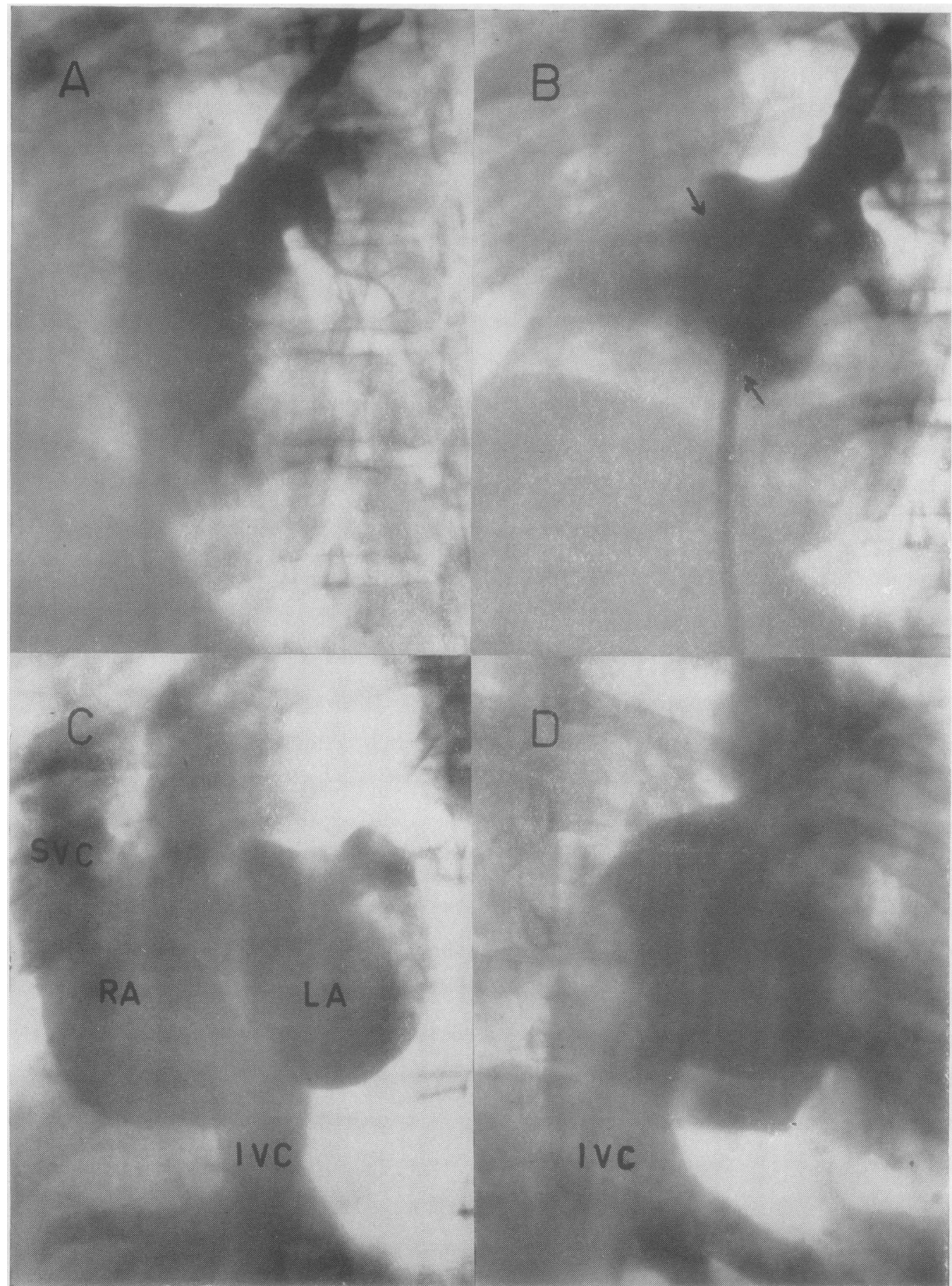

FIG. 1.-Demonstration of an atrial septal defect measuring $4 \frac{1}{2} \mathrm{~cm}$. in diameter and displacement of the inferior vena cava. Early stage of contrast-filling of the left atrium (A). The contrast passes into the right atrium (B) outlining the edges of the defect (arrows). There is a considerable reflux of contrast material into the inferior and superior vena cava. The inferior vena cava is displaced more medially (C) and posteriorly (D) than is usual. SVC=superior vena cava. IVC=inferior vena cava. $\mathbf{R A}=$ right atrium. $\mathbf{L A}=$ atrium. 


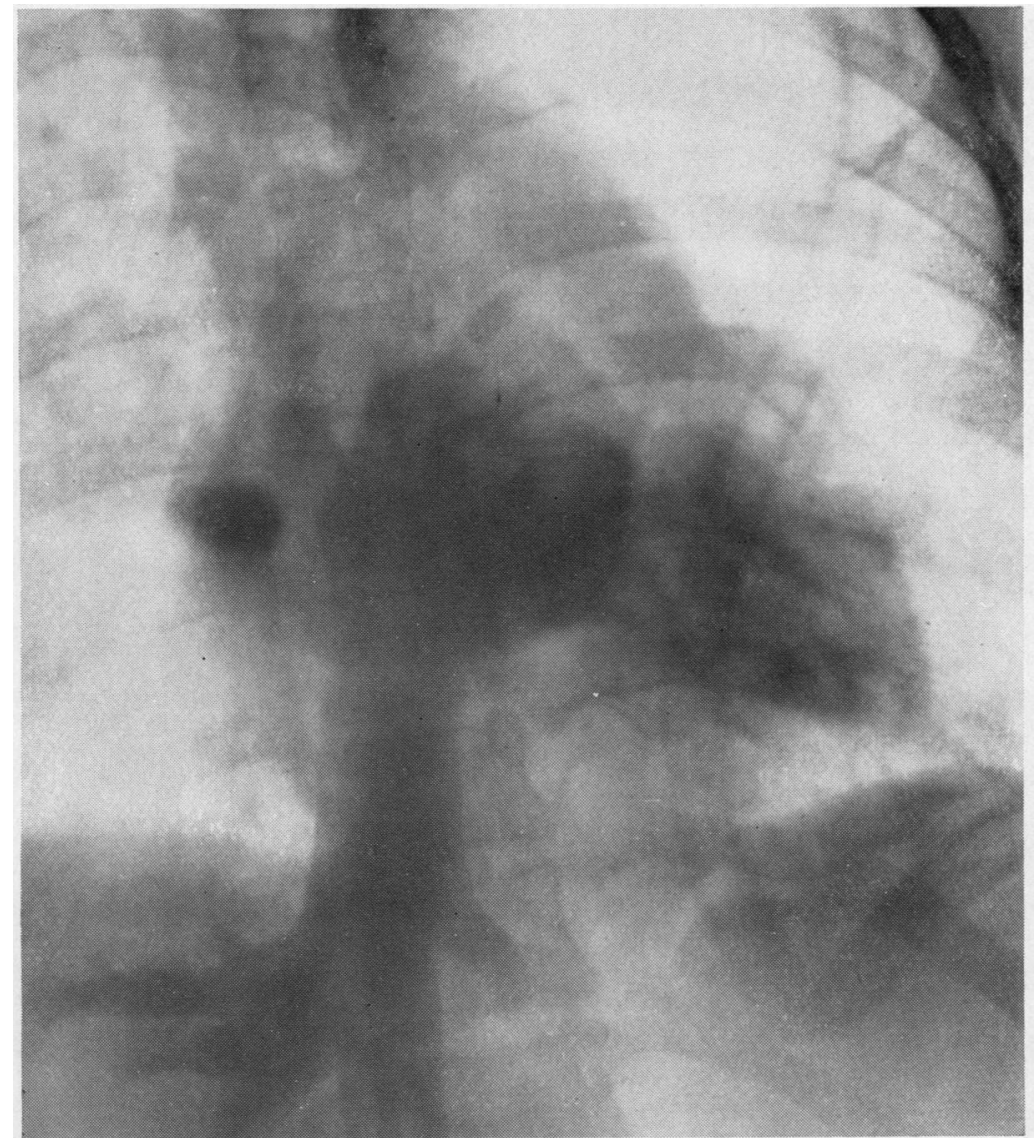

FIG. 2.-Angiocardiography with contrast injection into the inferior vena cava. The contrast material passes from the vena cava directly into the left atrium.

usually found when the defect is low and near to the orifice of the inferior vena cava (Fig. 1).

Operation was performed using hypothermia. The patient was immersed in ice water until a temperature of $33.4^{\circ} \mathrm{C}$. in the oesophagus was reached, resulting in a temperature of $28.5^{\circ} \mathrm{C}$. at the inflow-occlusion. The $\mathrm{pCO}_{2}$ and the $p \mathrm{H}$ were checked during hypothermia every five to 10 minutes and ventilation adjusted to $p \mathrm{H} 7.55$ to 7.6. The inferior and superior vena cava and the pulmonary veins from both lungs were occluded and after 30 seconds a clamp was applied over the root of the aorta and $25 \mathrm{mg}$. of prostigmine was injected proximal to the clamp. The clamp occluding the incision which had already been made in the right atrium was opened and the atrial septal defect sutured. When the defect had been closed the surgeon tried to pass his finger into the inferior vena cava, but the occluding tape around the vessel had been placed too close to the right atrial wall to make it possible to see or palpate the first part of the inferior vena cava. The right atrium was then filled with saline solution and a clamp applied to close the incision. The clamps were then released from the aorta and from the superior vena cava and from the pulmonary veins on both sides. A little later the clamp was taken off the inferior vena cava. The heart resumed its work well, but after five minutes it went into ventricular fibrillation. Heart massage was instituted, but an extraordinary sensation was experienced because the left ventricle seemed to have a good tonus and filled well so that it was easy to keep up a eripheral systemic blood pressure of over $100 \mathrm{~mm}$. Hg. The big right ventricle seemed to be empty and llaccid. It was not possible to defibrillate the heart with an electric shock, probably due to the flaccid empty right ventricle. Heart massage was continued for one hour when the heart was defibrillated chemically with 25 times concentrated Ringer's lactate of which $3 \mathrm{ml}$. was injected directly into the left ventricle. Also $3 \mathrm{ml}$. of $10 \%$ sodium lactate was injected into the left ventricle and the heart massage continued. A few minutes after the last dose of lactate the heart resumed a normal sinus rhythm. The incision was closed and the patient was rewarmed in water at $45^{\circ} \mathrm{C}$. in a bath tub. 


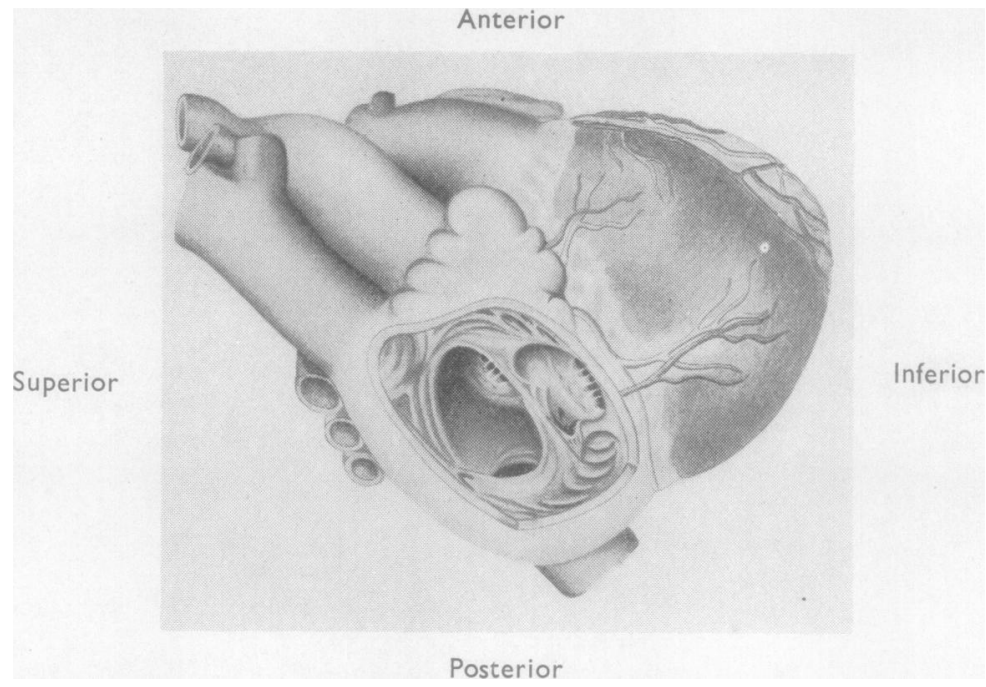

FIG. 3.-Diagram from our case with a big atrial septal defect of the secundum type. Anteriorly the defect has an inferior edge of atrial septum, which continues with the valve of the inferior vena cava.
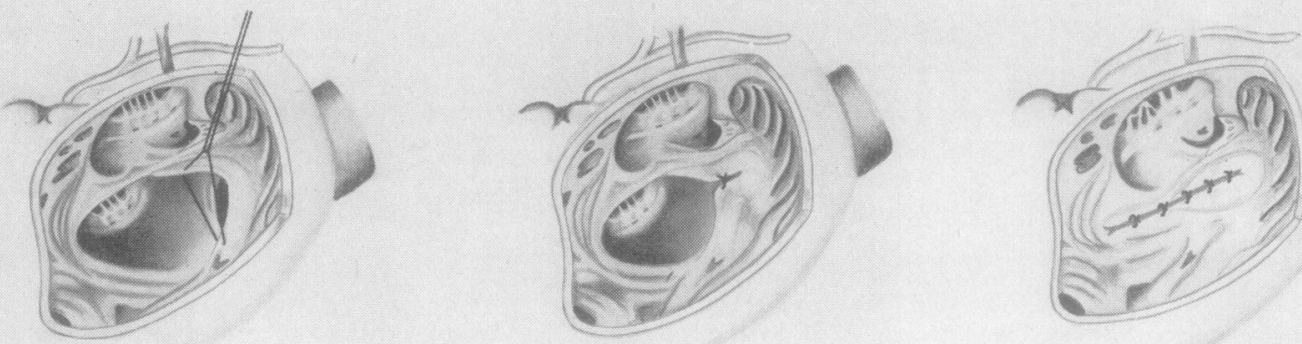

FIG. 4 A, B, C.-When the valve of the inferior vena cava was wrongly taken as the lower margin of the defect and used for repair of the defect by direct suture, the inferior vena cava was connected to the left atrium and not to the right (cf. Fig. 2).

Fig. 5 A, B, C.-After removal of the first suture a new closure was made and the lowest stitch consisted of several bites taken in the lowest part of the left atrial wall and tied on the left side on the inferior vena cava before the rest of the defect was closed. Thus the orifice of $\sigma$ the inferior vena cava was forced to open into the right atrium. 
The patient was very cyanotic, and arterial puncture showed an oxygen saturation of only $68 \%$. Tracheostomy was performed and the patient ventilated with a respirator. Radiological examination of the lungs showed no atelectasis, but the patient continued to be extremely cyanotic and it was suspected that the inferior vena cava had been diverted into the left atrium. A catheter was again introduced from a saphenous vein up into the inferior vena cava, but it entered the left atrium directly. The oxygen saturation was $49 \%$ in the inferior vena cava, $78 \%$ in the left atrium, $75 \%$ in the brachial artery, and $98 \%$ in a pulmonary vein. This investigation showed a very big right-left shunt at the atrial level. To visualize the anatomy an angiocardiograph was made with contrast injection through the catheter in the inferior vena cava (Fig. 2), when it was found that the inferior vena cava drained directly into the left atrium. The catheter was again introduced into the left atrium and left there.

The patient was again immersed in an ice-bath, and when the oesophageal temperature was $28.5^{\circ} \mathrm{C}$. inflowocclusion was again performed and the incision into the right atrium was opened. The patient had now become acidotic and needed bicarbonate and hyperventilation at $20 \mathrm{l} . / \mathrm{min}$. before the inflow-occlusion could be performed.

After occlusion of both the vena cava and the lung veins the incision was opened into the right atrium and the suture in the atrial septum was taken out. The catheter that had earlier been pushed up into the left atrium was grasped and lifted out into the wound so that the posterior aspect of the orifice to the inferior vena cava could be visualized. A new closure was made, and the lowest stitch, consisting of several bites taken in the lowest part of the left atrial wall, was placed and tied on the left side of the inferior vena cava before the rest of the defect was closed by a running suture (Fig. 5). The orifice of the inferior vena cava now opened into the right atrium. Before the last suture was tied the left atrium was filled with saline and the occlusion on the pulmonary veins was released. The right atrium was then filled with saline solution. The clamp was placed over the incision and the occlusion to the superior vena cava was released to fill the right atrium with blood. This second occlusion was of six minutes' duration. The wound was re-sutured and the patient placed in the warm water-bath. Respirator treatment was then continued post-operatively. Immediately after operation the patient showed paralysis of the right arm and the right leg; but these symptoms disappeared within 14 days. Arterial saturation was $92 \%$ one month after the operation. Six months later arterial saturation was $65 \%$ and a residual shunt was found.

Eight months after the operation the patient was re-examined. Subjectively she had considerably improved, but the exercise tolerance test showed a physical working capacity of only $250 \mathrm{~kg}$. $/ \mathrm{min}$., which was considerably lower than before the operation. The arterial oxygen saturation at rest in repose was only $88 \%$. A new examination with cardiac catheterization and angiocardiography was then performed. The

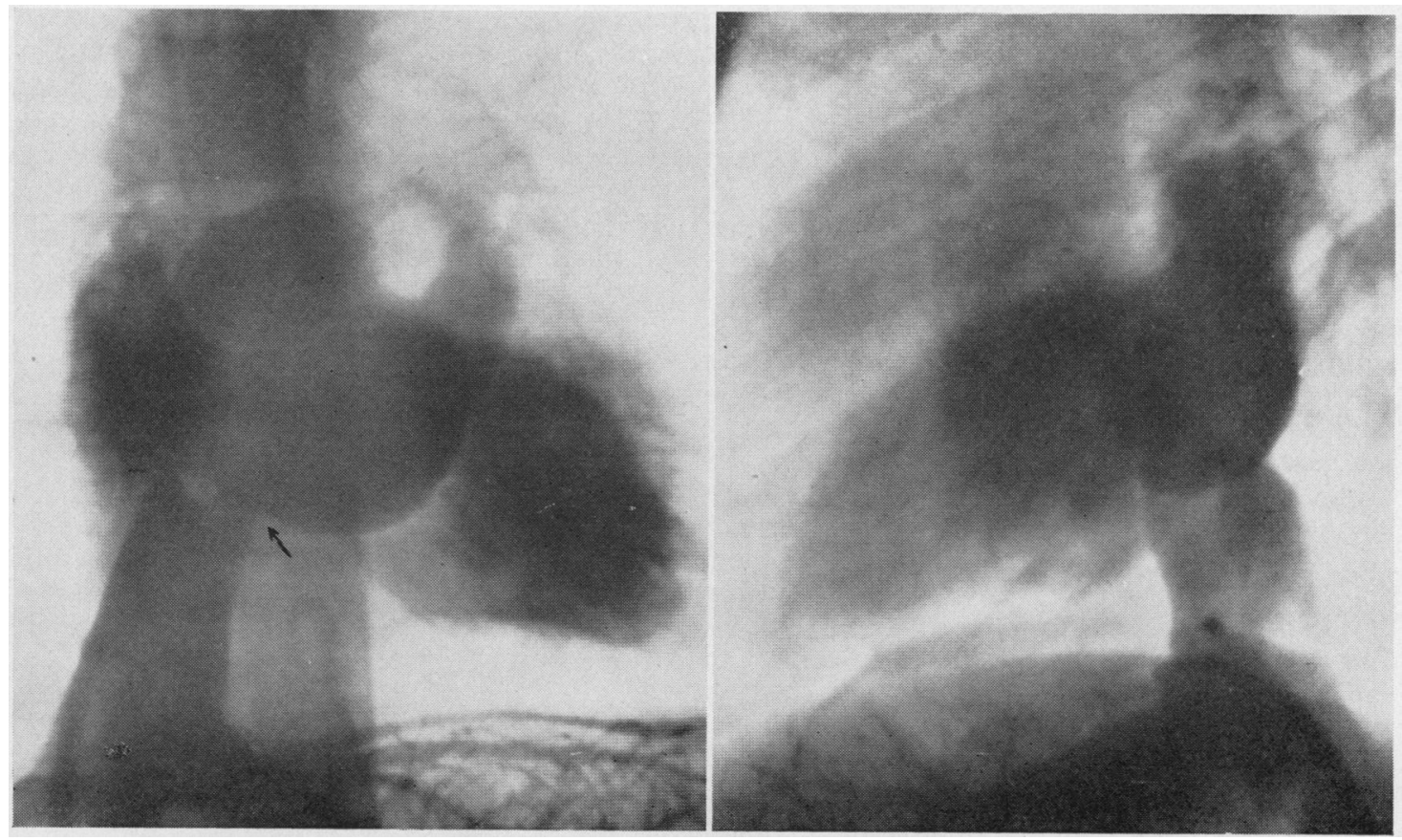

FIG. 6.-Angiocardiograph eight months after operation with contrast injection into the inferior vena cava. The contrast material passes not only to the right but also into the left atrium. Place of leakage is indicated by arrow. 
catheter was introduced from the right groin and could be passed from the inferior vena cava to the right as well as to the left atrium. There was no left-right shunt $\left(\mathrm{O}_{2}\right.$ saturation in the superior vena cava $=67 \%$, in the inferior vena cava $=68 \%$, and in the pulmonary artery $64 \%$ ), but on the other hand there was a right-left shunt from the inferior vena cava to the left atrium $\left(\mathrm{O}_{2}\right.$ saturation in the pulmonary vein $=98 \%$ and in the brachial artery $=88 \%$ ). The communication between the right and left atrium was demonstrated by an injection of contrast medium into the inferior vena cava (Fig. 6). A suture must have cut through causing this leakage through the suture line. To prevent this we are now always placing two to four isolated sutures after the continuous ones have been inserted.

\section{Discussion}

The surgeon operating on an atrial septal defect must always have in mind the possibility that the inferior edge of the defect is the valve of the inferior vena cava. In this case the surgeon thought of this possibility and yet he failed to prove the anatomy. The error could have been avoided if the two following precautions had been taken:

(1) If the surgeon had first put his index finger through the right auricular appendage for a thorough palpation of the inferior of the right atrium, he would have detected the valve of the inferior vena cava. As it was, he felt a big atrial septal defect through the wall of the right atrium. An exact anatomical picture of the right atrium can be obtained by a palpation through the auricular appendage.

(2) The occlusion tape around the inferior vena cava was placed too close to the right atrium so that it was difficult to see or palpate the first part of the inferior vena cava once the atrium had been opened. If the occlusion tape on the inferior vena cava can be placed as far away as possible from the right atrium it is easier to locate its orifice from the inside of the right atrium. If one still has any doubt about the anatomy it is advisable to release the occlusion on the inferior vena cava for a monent to see where the blood enters the heart from this vessel. Swan, Wilkinson, and Blount (1958) have in one case found it necessary to excise the valve of the inferior vena cava and then actually to transplant the inferior vena cava to the right side before it was possible to close the atrial septal defect. This required two separate caval occlusions. Once the anatomy is clearly understood it is easy to make a new orifice for the inferior vena cava inside the right atrium by placing one low stitch taking several bites in the lower part of the left atrial wall so as to form the medial half-circle of the inferior vena cava orifice. From this point a running suture through the edges of an atrial septal defect can be carried to the upper corner of the defect.

If the error is made of diverting the inferior vena cava into the left atrium it is absolutely necessary to reoperate upon the patient as soon as possible, and at the reoperation the suture in the atrial septal defect must be taken away and a new one placed in the correct position so that the orifice of the inferior vena cava opens into the right atrium. We know cases where this has been tried $a_{i}$ the second inflow-occlusion during the same operation without success. If this is done it is important to wait long enough before the second occlusion is performed. In our case, where it was necessary to massage the heart for one hour before it reverted to sinus rhythm, we considered it advisable to warm the patient first and by waiting a little to secure temporarily an efficient circulation utilizing artificial ventilation with the respirator. In this way the arterial oxygen saturation did not increase to more than $78 \%$ and the patient deteriorated. It was necessary therefore to induce hypothermia once more the same day to lower the oxygen requirements and to proceed as soon as possible with the reoperation. We know that one patient has been left with an inferior vena cava draining into the left atrium for two days ; but that patient died. It seemed to us important to give the patient a long enough rest after the first occlusion but not to wait too long before operating again.

The management in our case seems to have been favourable. The difficulty of defibrillating the heart in the ordinary way by an electric shock when there was an empty and flaccid right ventricle was obvious. The good effect of a high concentration of lactate solution injected directly into the left ventricle was demonstrated.

\section{SUMMARY}

A case of atrial septal defect is described where the inferior vena cava seemed to enter the left atrium. There was a big valve of the inferior vena cava which was erroneously used to repair the defect and this resulted in a diversion of the inferior vena caval blood into the left atrium.

The successful management of this çase at reoperation during hypothermia for the second time on the same day is described.

\section{REFERENCES}

Bedford, D. E., Sellors, T. H., Somerville, W., Belcher, J. R., and Besterman, E. M. M. (1957). Lancet, 1, 1255.

Lowis, F. J., Varco, R. L., and Taufic, M. (1954). Surgery, 36, 538. Swan, H., Wilkinson, R. H., and Blount, S. G.(1958). J.thorac. Surg., 35, 139. 